\title{
MedienPädagogik
}

Zeitschrift für Theorie und Praxis der Medienbildung

Jahrbuch Medienpädagogik 16:

Medienpädagogik in Zeiten einer tiefgreifenden Mediatisierung

Herausgegeben von Karsten D. Wolf, Klaus Rummler,

Patrick Bettinger und Sandra Aßmann

\section{Die vernetzte Schulklasse}

\section{Exploration zu Konstruktionen individueller und kollektiver Lernaktivitäten am Beispiel von WhatsApp- Gruppenchats}

\author{
Caroline Grabensteiner
}

\section{Zusammenfassung}

Wie organisieren Schülerinnen und Schüler ihre Lernaktivitäten in einer Welt, die von tiefgreifender Mediatisierung geprägt ist? Welche Rolle spielen Kommunikationsplattformen und ihre vielfältigen Möglichkeiten der Vernetzung ausserhalb der Schule für Hausaufgaben und häusliche Lernaktivitäten? Diese Fragen zielen auf das Medienhandeln als konstruierenden und konstruierten Kontext. Versteht man Schulklassen als kommunikative Figurationen, so lässt sich diese spezifische Akteurskonstellation und ihr Repertoire an Medien untersuchen; dieses Repertoire strukturiert Kommunikation einerseits und befähigt andererseits zur individuellen und kollektiven Aushandlung des thematischen Rahmens. Innerhalb dieser Formationen lassen sich Handlungs- und Wirksamkeitsräume identifizieren, die Bildungspotentiale beinhalten. Der folgende Beitrag möchte anhand ausgewählter Beispiele aus einer Studie im Mixed-Methods Design Licht in ausserschulische Lernaktivitäten bringen. Dabei werden Ansätze der Sozialen Netzwerkanalyse (SNA) mit interpretativen Methoden der Datenauswertung kombiniert. Ergebnis dieser Methodenkombination ist eine Darstellungsform zur Rekonstruktion des Zusammenspiels zwischen individuellem und kollektivem Medienhandeln in Bezug auf Lernaktivitäten. 


\title{
Networking School Classes. Approaching constructions of individual and collective learning activities at the example of WhatsApp-chatgroups
}

\begin{abstract}
How do students organise their learning activities in a world shaped by deep mediatisation? How do they appropriate media into their daily learning practice? How do communication platforms facilitate networking in out-of-schoolcontexts through manifold possibilities for communication during domestic learning activities? These questions target media activities in school classes as practices of constructing and constructed contexts. Looking at school classes as communicative figurations helps to understand the specific constellation of actors, accessing a certain repertoire of media. This appropriation structures as well as enables individual and collective frames and settings for communication. This article aims to give insight into out-of-school working-contexts of pupils. For this purpose, selected findings of a mixed methods study will be discussed, combining social network analysis (SNA) and interpretative data analysis to give a relational view on the (re)construction of collective and individual working contexts.
\end{abstract}

\section{Einleitung}

"In some respects, "the class" is a meaningful unit; in other respects, it is a figure of convenience.» (Livingstone und Sefton-Green 2016, 61).

Eine Schulklasse ist eine konstruierte und konstruierende Einheit: Sie ist sowohl Ausdruck sozialer Konventionen als auch Ort kommunikativer Bedeutungskonstitution. Unter dem Vorzeichen von Prozessen tiefgreifender Mediatisierung (Krotz und Hepp 2012; Hepp 2018) sind die Kommunikationsformen, die Schülerinnen und Schüler individuell und kollektiv innerhalb ihrer alltäglichen Lernaktivitäten herstellen, unter Einbezug medialer Entwicklungen zu diskutieren. Konkret geht es darum, wie die Möglichkeiten zu schulbezogener Kommunikation sich auf Lernaktivitäten 
ausserhalb des Schulgebäudes auswirken. Zum Beispiel beschäftigt die Kooperation zu, oder das Abschreiben von Hausaufgaben Eltern und Lehrpersonen gleichermassen. Die Frage, wie digitale Medien in die schulische Zusammenarbeit integriert werden, welche sozialen Strukturen innerhalb der Klasse wirksam sind und welche Potenziale dies für ein Verständnis von Medienbildung hat, soll im folgenden Beitrag anhand konkreter Beispiele diskutiert werden.

Als einen Ausschnitt aus dem vielfältigen Medienensemble, das Schülerinnen und Schülern zur Verfügung steht, sind die aktuell immer populärer werdenden Instant-Messaging Dienste im Fokus. Die Plattform WhatsApp hat sich in den letzten Jahren durchgesetzt und ist mit einer Nutzungsbreite von $87 \%$ beliebtestes Kommunikationsmittel der 12-19-Jährigen am Smartphone (Feierabend, Rathgeb, und Reutter 2018, 36). Nach YouTube nimmt die App demnach den zweitwichtigsten Platz unter den Internetanwendungen ein. Diese Ergebnisse können für Schweizer Jugendliche anhand der Ergebnisse der Repräsentativstudie JAMES (Suter u. a. 2018) bestätigt werden. Zur Nutzung unterschiedlicher Handyfunktionen von Nachrichtendiensten, über Wecker und Kalender bis hin zu Informationsanwendungen gaben 95\% der Jugendlichen im Alter zwischen 12 und 19 Jahren an, mehrmals pro Woche, bzw. 88\% täglich einen Messenger-Dienst, wie z. B. WhatsApp zu nutzen, um sich mit anderen auszutauschen. Von den Messenger-Nutzern gaben 89\% an, auch regelmässig den Austausch in Gruppen mithilfe der Gruppenchatfunktion (Suter u. a. 2018, 66) zu pflegen. Instant Messaging bezeichnet in zu diesem Zweck bereitgestellten Online-Räumen erfolgende textbasierte Kommunikation, die mit multimedialen Inhalten und externen Verlinkungen ergänzt werden kann und sowohl zeitlich als auch räumlich unabhängig ist. Sowohl die Eins-zuEins- als auch die Gruppenkommunikation werden unterstützt. Jugendliche beziehen diese Kommunikationsformen in informelle, schulbezogene Lernkontexte mit ein. Wie genau sich diese Form des Medienhandelns entwickelt, welche sozialen, kulturellen und gesellschaftlichen Strukturen dabei (re)produziert werden, welche Handlungs- und Wirksamkeitskompetenzen Schülerinnen und Schüler dabei ausbilden und welche Potentiale für Medienbildung sich darin konstituieren, ist noch nicht hinreichend erforscht. 
Dieser Beitrag untersucht Medienhandeln als kommunikative und soziale Formation, innerhalb derer sich individuelle und kollektive (Medien-) Bildungsverhältnisse konstituieren. Als Grundlage werden strukturelle Eigenschaften sozialer Konstellationen als Voraussetzung für Handlungsund Wirksamkeitskompetenz analysiert. Die Frage nach Agency nimmt Bezug auf die Einbettung individueller Handlungszusammenhänge innerhalb dieser Strukturen. Schülerinnen und Schüler stehen im Beziehungsgefüge von Hausaufgaben als schulischer Lernkontext bzw. alltäglichem Medienhandeln und sozialen, gesellschaftlichen, sowie technologischen Strukturen (Seipold, Rummler, und Rasche 2010). Es geht darum, diese Zusammenhänge so darzustellen und analytisch zu durchdringen, dass der Komplexität Rechnung getragen und Anteile individueller und kollektiver Leistungen zu seiner Konstitution identifiziert werden können. Daraus ergeben sich drei analytische Bestandteile, die in einer übergeordnete Forschungsfrage zusammengefasst werden:

- Wie konstituiert sich Medienbildung im vernetzten Medienhandeln in ausserschulischen Lernkontexten?

Aus dem Gegenstand ergeben sich drei Teilfragen, die jeweils auf unterschiedliche Dimensionen der Analyse zielen:

- Welche Akteurskonstellationen, thematischen Rahmungen und Kommunikationsformen konstituieren Schulklassen als kommunikative Figurationen?

- Wie entfaltet sich innerhalb dieser Formationen individuelle und kollektive Handlungsfähigkeit im Sinne von «Agency» als Handlungsdimension?

- Wie lässt sich ein relationales Bildungsverhältnis innerhalb dieser Strukturen rekonstruieren?

Instant Messaging Dienste bieten die Möglichkeit zur unmittelbaren und/oder nachvollziehenden Teilhabe an Gruppenaktivitäten. Damit sind sie integrativer Teil der kommunikativen Konstruktion sozialer Gefüge. In Bezug auf WhatsApp-Kommunikation in Schulklassen haben sich innerhalb der Erziehungswissenschaft unterschiedliche Forschungsfelder etabliert. Einerseits werden Gruppenchats und die Auswirkungen auf 
Sprachkompetenz untersucht (Gimranova, Nurmanova, und CohenMiller 2017; Hayes und Mansour 2017; Keogh 2017; u.a. Avci und Adiguzel 2017). Andererseits wird WhatsApp als «natürliches» Learning Management kontrastierend $\mathrm{zu}$ institutionalisierten Lernplattformen dargestellt (So 2016; Cetinkaya 2017a; 2017b; Jannari u. a. 2017). Einige Studien untersuchen die Kommunikation von Schülerinnen und Schülern mit Lehrpersonen in WhatsApp und deren Auswirkungen auf den Schulalltag (u.a. Bouhnik und Deshen 2014; Rosenberg und Asterhan 2018). Das Thema «sharing», also das Teilen von Wissensinhalten bei jüngeren Altersgruppen ohne Beteiligung von Lehrpersonen, ist ein noch wenig bearbeitetes Forschungsfeld. Asterhan und Bouton untersuchten Motive von Schülerinnen und Schülern im Alter von 14-19 Jahren für das Teilen von Wissen im Klassenverband. Dabei lag der Fokus darauf, welche persönlichen Vorteile - «benefits»; (Asterhan und Bouton 2017, 21) - sich die Teilnehmenden davon versprechen. Unklar bleibt, wie die soziale Dynamik in der Schulklasse das Teilen von Wissen beeinflusst, wer mit wem teilt und welche sozialen und kommunikativen Aktivitäten und Dynamiken in Schulklassen wirksam sind und ausverhandelt werden. Diese Fragen greift der vorliegende Artikel mit einem erweiterten Blick auf Lernaktivitäten auf.

Als erstes sollen überblicksartig sensibilisierende Konzepte zur theoretischen Verortung des Projekts vorgestellt werden. Darauf folgt die Darstellung des Mixed Methods Ansatzes sowie die Analyse anhand ausgewählter Beispiele.

\section{Sensibilisierende Konzepte - relationale Perspektive}

Das Folgende stellt kein abgeschlossenes Theoriekonstrukt, sondern «interpretive framewoks» (Charmaz und Belgrave 2015, 2) als einen integrativen Bestandteil der theoriegenerierenden Vorgehensweise dar. Die Konzepte stellen als Vorwissen Weichen für Entscheidungen auf dem Weg der Datenanalyse. Sie sind somit Interpretationsrahmen für die Annäherung an soziale Wirklichkeiten. Drei Metakonzepte bilden auf unterschiedliche Ebenen die analytische Suchbewegung ab. Auf Ebene der kommunikativmedialen Struktur soll der Ansatz der kommunikativen Figurationen vorgestellt werden. Ausgehend von einer Beschreibung und Analyse sozialer 
und kommunikativer Strukturen kann auf Ebene des Medienhandelns weiter die Frage nach relationaler Handlungs- und Wirksamkeitskompetenz und weiters nach Bildung als reflexives Verhältnis aufgeworfen werden.

\subsection{Kommunikative Figurationen - mediale Relationen}

In Weiterentwicklung der Mediatisierungstheorie (Krotz und Hepp 2012; Hepp und Krotz 2012; Krotz 2007) und in Bezugnahme auf die Netzwerktheorie Emanuel Castels (2004), sowie das Konzept der Figurationen nach Norbert Elias (1999) entwickelte die internationale Arbeitsgruppe «Communicative Figurations» (Hepp, Breiter, und Hasebrink 2018) das Theoriekonzept der «tiefgreifenden Mediatisierung» (Hepp 2018). Kommunikative Figurationen bieten darin einen Analyserahmen, um die vielfältigen «Verflechtungszusammenhänge» (Hepp 2013; Hepp und Hasebrink 2014) kommunikativer und medialer Entwicklungen empirisch fassen zu können. Darunter werden «musterhafte Interdependenzgeflechte von Kommunikation» verstanden, «die über verschiedene Medien hinweg bestehen und auf eine bestimmte 〈thematische Rahmung〉 ausgerichtet sind, an der sich das kommunikative Handeln orientiert» (Hepp 2013, 85; Hepp und Hasebrink 2014, 351). Auf dieser Basis können unterschiedliche Akteursformationen, ihr Handeln mit medialen Artefakten, dem eine Regelhaftigkeit sowie kontextuelle und soziale Verortung zugrunde liegt, beschrieben werden. Es bietet die Möglichkeit, die Relationalität von Medien integrativ im Handeln zu verorten. So verstanden sind sie nicht Vermittlerinnen, im Dienste von Kommunikation, sondern Teil der Sinn- und Bedeutungskonstruktion.

Zur Beschreibung des Medienhandelns formulieren Hepp und Hasebrink folgende Aspekte:

- Erstens können Kommunikationsformen als «regelhafte Weisen kommunikativen Handelns, die sich zu komplexeren Mustern kommunikativer Praxis fügen» (Hepp und Hasebrink 2014, 354) verstanden werden. Es geht um die Art und Weise wie Kommunikation an soziale, kulturelle und technologische Regelhaftigkeiten gebunden ist.

- Zweitens soll das Medienensemble als alle medialen Formen beschrieben werden, die für eine bestimmte kommunikative Figuration typisch sind. 
- Drittens ist die spezifische Akteurskonstellation zu identifizieren, die alle Akteursebenen vom Individuum, über Institutionen und soziale Gruppierungen bis zur Gesamtheit der globalisierten Gemeinschaft umfassen kann. (Hepp 2013, 87f).

- Als vierten Punkt arbeiten Hepp und Hasebrink die thematische Rahmung heraus, als «handlungsleitendes und orientierendes Thema der kommunikativen Figuration, das diese als ein sinnhaftes (Ganzes〉 erfassbar macht» (Hepp und Hasebrink 2014, 354) kann sie sowohl inhaltliche Orientierung, als auch kulturelle, soziale und technologische Rahmen abbilden.

Schulklassen als kommunikative Figurationen zu verstehen macht im medienpädagogischen Kontext insofern Sinn, als dieser Analyserahmen einerseits eine Interpretationsfolie für die einzelnen Komponenten des untersuchten Settings und andererseits genug Offenheit für ein exploratives Vorgehen bietet. Ausgehend davon können Schulklassen und ihr kommunikatives Handeln empirisch erfasst und die vier Ebenen als ineinandergreifende Elemente verstanden werden. Der Ansatz eignet sich speziell zur Annäherung an die strukturelle Verfasstheit medial-kommunikativer Formationen. Schulklassen können vor diesem Hintergrund als von tiefgreifender Mediatisierung geprägte, transmedial und translokal verortete Akteurskonstellationen beschrieben werden. Anhand von Instant-Messaging Gruppen als Ausschnitt aus dem Medienensemble werden die thematische Rahmung und die Kommunikationsformen Jugendlicher in ihrer schulbezogenen Kommunikation untersucht. So können Themen, Zugehörigkeiten und Gruppenbildung, sowie das Zusammenspiel individueller und kollektiver Handlungs- und Artikulationsformen diskutiert werden.

Ausgehend von der medial-relationalen Struktur wird weiter nach $\mathrm{Me}$ dienhandeln gefragt, das innerhalb dieser Figurationen situiert und konstituiert ist. Das Modell von Struktur und Agency bildet den theoretischen Rahmen für ein Verständnis des Verhältnisses individueller und kollektiver (Medien)Handlungszusammenhänge. 


\subsection{Agency - Handlungsrelationen}

Individuelles Handeln und seine strukturelle Eingebundenheit in gesellschaftliche, kulturelle, soziale, usw. Strukturen wird im Konzept von Agency aufgegriffen. Innerhalb dieses Theoriekomplexes soll aus medienpädagogischer Perspektive die Frage nach «Handlungs- und Wirksamkeitskompetenzen» (Rummler 2018, 146) gestellt werden. Dies beinhaltet die Untersuchung, «wie es Lernenden [...] gelingt, ihre Lebenswelt reflexiv und verantwortlich mit Medien zu konstruieren und aufzubauen, sowie [...] Teilhabe» an sozialen und kulturellen Handlungszusammenhängen zu erlangen und somit kompetent «auf Welt handelnd einzuwirken» (ebd.). Diese Perspektive auf Medienhandeln im Spiegel relationaler Agency soll im Folgenden hergeleitet werden.

\subsubsection{Handelnde Auseinandersetzung mit Welt}

Ausgegangen wird von Giddens' Konzept der «knowledgeability» (1984, 21f) als Verortung individuellen Handelns in Wissensstrukturen um Regeln und Ritualisierungen die ihm eingeschrieben sind und zugleich durch jede einzelne Handlung konstituiert werden. Diese Dynamik ist als «duality of structure» (Giddens 1984, 29) Voraussetzung der Analyse relationaler Handlungskonstitution. Im deutschsprachigen Diskurs rezipierte vor allem die «Mobile Learning Group» (Pachler, Bachmair, und Cook 2010; Bachmair 2009; Bachmair, Cook, und Pachler 2014) das Agency-Konzept. Das Dreieck aus «Agency», Strukturen und kulturellen Praktiken (Pachler, Bachmair, und Cook 2010, 25) stellt das (Bildungs-)Subjekt in eine Beziehung zu seiner materiellen und sozio-kulturellen Umwelt, als Bezugnahme auf das Selbst- und Weltverhältnis des im nächsten Punkt ausformulierten Bildungsverständnisses.

Margret Archer arbeitet das «social self» (Archer 2000, 254) als individuell motiviertes, handelndes und humanes, sowie emotionales interplay (Archer 2000, 307) von Struktur und Subjekt heraus. Sie argumentiert für ein in seiner Reflexionskraft «starkes» Subjekt bzw. Individuum. Es ist in der Auseinandersetzung mit «Agency» als Konzept die Entwicklung des Begriffs und auch die kritische Wendung gegen Giddens und Archer - vor allem in Form der Subjektkritik - zu beachten. 


\subsubsection{Agency - Action - Structure}

Mustafa Emirbayer und Anne Mische (1998) formulieren Agency als temporale Konstruktion, als Zusammenspiel von Iteration (Vergangenheit), Projektion (Zukunft) und Evaluation (Gegenwart). Sie sehen die Triade aufgespannt zwischen Agency, als Handlungspotential, Action im Sinne konkreter Handlung innerhalb eines zeitlichen Kontext, der Struktur als Konstruktionszusammenhang (Emirbayer und Mische 1998, 1003). Die analytische Trennung von Handlung und Handlungskompetenz greift Ian Burkitt auf, der das Konzept der «Relational Agency» herausarbeitet. Ihm geht es weniger darum, «past agency of individuals» (Burkitt 2018, 529) zu rekonstruieren, also im Nachhinein temporale Strukturen, oder reflexive Wendungen in Handlungen aufzusuchen, sondern Handeln auf seine relationale Konstitution zu untersuchen:

«Agency appears only among people in their relational contexts» (Burkitt 2015, 332).

In diesem Verständnis wird der Handlungszusammenhang in seiner strukturellen Gegebenheit als Kontext aufgespannt und die konkrete Handlung in ihre Zusammenhänge gestellt. Eine analytische Trennung zu implizieren, die mithilfe einer reflexiven Wendung wieder aufzulösen ist, sieht Burkitt nicht vor. Auf der Ebene der empirischen Annäherung an individuelles und kollektives Handeln ist die «meaningful formation of relationships and the identities within them» (Burkitt 2015, 332) als erster Analyseschritt sinnvoll. Dies drückt sich in der thematischen Rahmung der kommunikativen Figurationen aus und stellt diese in ihren kollektiven Aushandlungskontext.

Um von der Handlungsebene auf Bildungsprozesse zu abstrahieren, sind Rückbezüge auf die von Archer betonte Reflexivität und die räumlichzeitlichen kontextualen Verortungen bei Emirbayer und Mische sinnvoll. Burkitt macht das relationale Element von Agency sichtbar. Dies verhilft dazu, den Bildungsprozess nicht allein im sich bildenden Subjekt zu verorten, sondern auch kollektive Bildungsprozesse, die sich aus den individuellen Bildungsverhältnissen kultivieren, zu untersuchen. 


\subsection{Medienbildung - Bildungsrelationen}

Bildung wird klassischerweise als Verhältnis formuliert. Das relationale Moment muss insofern nicht eigens herausgearbeitet werden. Aus der oben verhandelten Perspektive auf soziales und kommunikatives Medienhandeln ist es konsequent, das klassische Bildungsverhältnis bezogen auf Medien zu aktualisieren. Norbert Meder formuliert - in Anlehnung an Humboldt (Meder 2014) - Medienbildung als Konkretisierung von Bildung als dreifaches Verhältnis:

- des Einzelnen vermittelt über ein Medium zu den Sachen und Sachverhalten in der Welt (dem Dinglichen),

- des Einzelnen vermittelt über ein Medium zu dem oder den Anderen in der Gemeinschaft (dem Sozialen),

- des Einzelnen vermittelt über ein Medium zu sich selbst in Vergangenheit, Gegenwart und Zukunft in der Zeit (seines Lebens). (Meder 2007, 70; Hervorhebung im Original)

Bildung ist laut Meder einerseits der Prozess (Meder 2007, 70), andererseits das Resultat (Meder 2015, 3) dieser Triade. Damit können sowohl Entwicklung als auch Status des Bildungsverhältnisses analytisch dargestellt und für eine empirische Analyse operationalisiert werden.

Meder beschreibt Medienhandeln als «Vollzug in Raum und Zeit» (Meder 2007, 58), der aufgrund seiner Bindung an ein «raum-zeitliches Medium der Artikulation» (ebd.) an eine materielle Dimension, ein «Substrat» (Meder 2014, 64) gebunden ist. Beobachtbar sind diese Artikulationen in ihren sinnlich wahrnehmbaren Spuren. Darin liegt die Chance, die theoretisch dargestellten Begriffe im Medienhandeln aufzusuchen und nachzuvollziehen.

Eine Analyse der kollektiven und individuellen Bildungsverhältnisse vor dem Hintergrund ihrer medialen Vermitteltheit und Reflektiertheit als Artikulationen, bzw. als Reflexionsprozesse erfolgt in einem Dreischritt.

Kommunikative Figurationen bilden einen ersten Analyserahmen zur Annäherung an Strukturen und Akteurskonstellationen in ihren medialen und sozialen Verhältnissen, sowie den dahinter liegenden Konstruktionsprinzipien. 
Im Sinne von Medienbildung ist kommunikative Aushandlung integrativer Teil von Reflexionsprozessen und Projektions-/Reflexionsfläche für Bildungsverhältnisse. Auf dem Weg zu einer Annäherung an Bildungsprozesse werden die Voraussetzungen von Handlungs- und Wirksamkeitskompetenz in relationalen und medialen Repräsentationen des Medienhandelns identifiziert. Ziel ist es, mithilfe der analytischen Struktur Bildungspotenziale in Strukturen individueller und kollektiver Medien- und Lernaktivitäten zu rekonstruieren. Hierzu benötigt es ein Forschungsdesign, das in der Lage ist, die drei Komponenten Struktur, Handlungsdimension und Reflexion empirisch fassbar zu machen

\section{Bedeutungskonstitution in ihrem relationalen Zusammenhang rekonstruieren - ein Mixed Methods Design}

Die folgend dargestellte Analyse ist Teil der Entwicklung einer gegenstandsbezogenen «middle range theory»; (Charmaz und Belgrave 2015, 1) zu Medienbildung im vernetzten Medienhandeln von Schülerinnen und Schülern in informellen Lernkontexten. Die methodische Orientierung an Konzepten der Grounded Theory Methodology, versteht diese weniger als «Kochrezept» (vgl. Truschkat, Kaiser-Belz, und Volkmann 2011, 353), denn die Methoden der Datenerhebung und -auswertung im Mixed Methods Design folgen keinem klassischen Interview-Codierungs-Schema. Das konstruktive Element der Datengenerierung und seine Reflexion sind integrativer Bestandteil der kritischen Auseinandersetzung mit Datenauswertung als Interpretationsangebot (Charmaz 2011, 186). Anhand der gewählten Vorgehensweise zur Datenerhebung und -analyse entsteht ein datengetriebenes Theoriekonzept, das durch die Anpassung der Methoden an das Phänomen seine Erklärungskraft entfaltet. Eingedenk der sensibilisierenden Konzepte wird nach Formen der Verhandlung von relationaler Agency und Medienbildung innerhalb von Schulklassen als spezifische soziale Formationen gesucht. Die Konstruktion subjektiver und kollektiver Bedeutungszusammenhänge des Medienhandels in ausserschulischen Kontexten hält Herausforderungen für empirische Forschung bereit. Der Untersuchungsgegenstand löst sich von institutionellen und reicht in häusliche 
Bereiche hinein. Medienhandeln kann so oft nur indirekt beobachtet werden. Zum einen werden medial vermittelte Kommunikationskontexte, die räumlich nicht festlegbar sind, sondern sich in digitalen Sphären, auf persönlichen Endgeräten vollziehen, und zum anderen in geschlossenen Gruppen verhandelt. Dieser Ausgangslage wird mit einem explorativen Ansatz begegnet, die in Medienhandeln eingebettete Sinnkonstruktion von einer strukturellen über eine Handlungsebene hin zu einer reflexiven Ebene nachzuvollziehen.

Die Schulklasse wird, mit Bezug auf den oben dargelegten Analyserahmen, als Akteurskonstellation verstanden. Als soziale Gruppe setzt sie sich aus Individuen zusammen, die ihre je eigenen Praktiken - (regelbasiertes, routinisiertes Handeln) - Kontexte - (räumlich-soziale, sowie technische Verortungen und Voraussetzungen) - und thematischen Rahmungen (Anliegen, Absichten, Ziele) - einbringen. Durch die institutionelle Bindung innerhalb des Schulklassenprinzips sind sie strukturell kollektive Einheiten, die zu einer gemeinsamen kommunikativen Praxis, wie oben beschreiben, einer Verzahnung individueller und kollektiver Kommunikationsformen, finden. Dieser kollektive Handlungskontext ist strukturell in Schule verortet, was eine Einbettung in gesellschaftliche Kontexte mit sich bringt. Darüber hinaus haben klasseninterne, soziale Aushandlungsprozesse der Vergemeinschaftung und Individualisierung die Ausbildung weiterer, sozial-kommunikativ strukturierender Elemente zur Folge. Diese mehrfache Strukturierung ist aufgrund des Zwischenspiels (Archer 2000) von individuellen und kollektiven kontextualen, sozialen und kulturellen Voraussetzungen für jede Klasse neu zu hinterfragen. Schulklassen können als soziale Formationen charakterisiert werden die durch ihre Strukturierung aus gemeinsamen kulturellen und sozialen, institutionellen sowie gesellschaftlichen Regeln und Routinen erwachsen. Diese strukturellen Zusammenhänge darzustellen ist eine Herausforderung, der mit Methoden der Sozialen Netzwerkanalyse begegnet wird. Die Darstellung relationaler Daten gibt Hinweise auf strukturelle Zusammenhänge zwischen Akteurinnen und Akteuren. Ein spezieller Fokus der vorliegenden Untersuchung liegt in der Ermittlung des Zusammenhangs zwischen thematischen Rahmungen und Akteurskonstellationen, der sich mithilfe des Mixed Methods Designs aus Sozialer Netzwerkanalyse und interpretativen Methoden angenähert werden soll. 
Das analysierte Datenset stammt aus dem Projekt «Hausaufgaben und Medienbildung» (SNF Fördernummer 175 909, Rummler 2018). An der Erhebung nahmen Deutschschweizer Schülerinnen und Schüler der Sekundarstufe I im Alter von 11 bis 14 Jahren teil. Aus insgesamt 250 Medientagebüchern ${ }^{1}$ wurde mittels doppelten theoretischen Samplings das hier analysierte Datenset ausgewählt. Für eine Netzwerkanalyse ist die annähernde Vollständigkeit eines gesamten Klassensatzes notwendig, was in zwei Schulklassen der Fall war (Sekundarstufe I, 7. Schuljahr, max. 1 ein fehlendes Tagebuch pro Klasse). Im Sinne der Fallkontrastierung wurden für die Auswertung der Motive für die WhatsApp-Gruppen zwei Schulklassen anderer Schulstufen (Primarstufe, 6. Schuljahr, und Sekundarstufe I, 8. Schuljahr) mit ebenfalls möglichst grossem Rücklauf hinzugezogen. Die Netzwerkerhebung erfolgte im Rahmen einer im Tagebuch integrierten Netzwerkfrage. Fünf Klassenkolleginnen und -kollegen sollten für eine WhatsApp-Lerngruppe genannt werden. Insgesamt wurden bisher Netzwerkdaten von 46 Schülerinnen und Schülern als Gesamtnetzwerke von zwei Klassenverbänden ausgewertet. Für die Analyse der Motive liegen 79 Antworten vor. Diese Antworten stammen aus der offenen Frage: «Was würdet ihr in dieser Gruppe arbeiten?». Die interpretative Auswertung erfolgte über eine Wort-für-Wort Codierung, welche in die Ausbildung von vier Dimensionen mündete. Netzwerkdaten und Motive, werden in einer iterativ-vergleichenden Codierung zusammengeführt. Es folgt die Darstellung der Auswertung im Mixed-Methods-Design anhand ausgewählter Beispiele.

\subsection{Zentralität und Cluster - Ergebnisse der Sozialen Netzwerkanalyse}

«A network consists of a set of actors or nodes along with a set of ties of a specified type (such as friendship) that link them.» (Borgatti und Halgin 2011, 2)

1 Die Schülerinnen und Schüler dokumentierten vierzehn Tage lang ihre Medienaktivitäten während der Hausaufgaben in einem Journal-Heft (Rummler, Stingelin Schneider, und Grabensteiner 2018). 
Netzwerkdarstellungen bieten die Möglichkeit zur Analyse und Darstellung von Verbindungen zwischen Akteurinnen und Akteuren innerhalb sozialer Gruppen mithilfe graphentheoretischer Modelle. In Abb. 1 werden die wichtigsten in diesem Zusammenhang verwendeten Begriffe erklärt.

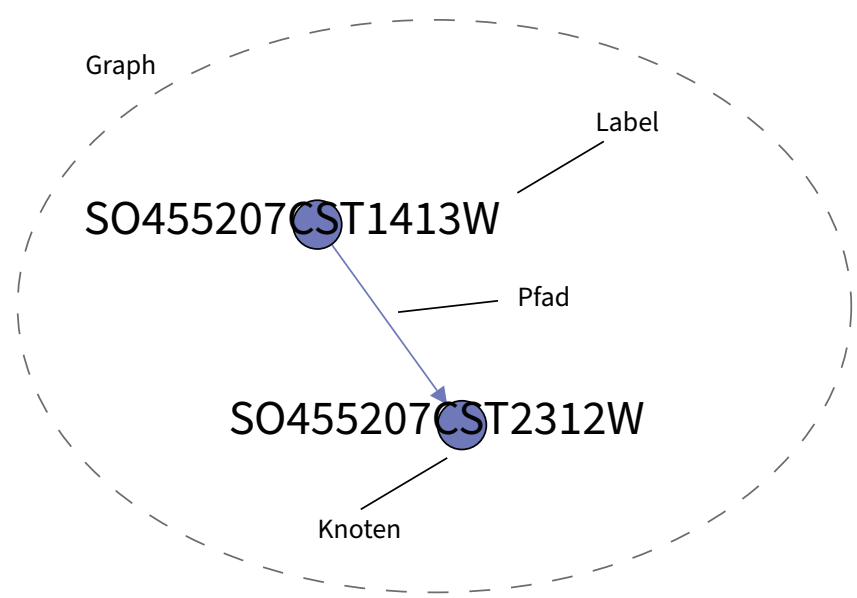

Abb. 1.: Netzwerkdarstellung (eigene Darstellung).

Bezogen auf das Datenset ist jedes Klassenmitglied durch einen «Knoten» repräsentiert. Die Labels (Knoten-Beschriftungen) ergeben sich aus der Anonymisierung (Klassencode: SO455207CST, letzte 5 Stellen: Code Schülerin/Schüler). Jede Nennung eines Klassenmitglieds für die Lerngruppe ist ein Pfad, bzw. Pfeil von einem zum anderen Knoten. Alle Knoten und Pfade ergeben ein Gesamtnetzwerk, das als Graph (Täube 2010, 397) bezeichnet wird. Abb. 1 zeigt einen Graphen mit zwei Knoten und einem Pfad ausgehend von SO455207CST1413W und eingehend zu SO455207CST2312W.

Aufgrund der gegebenen Optionen im Fragebogen können im vorliegenden Netzwerk von jedem Knoten maximal 5 Verbindungen ausgehen, während bei den eingehenden Verbindungen bis zu (n-1) Verbindungen möglich sind. Selbstnennungen wurden ausgeschlossen, mehr als 5 ausgehende Verbindungen wurden berücksichtigt. Auf Basis der Auswertung von «Zentralitäsmassen» (Borgatti, Everett, und Johnson 2018, 174ff) wird die Netzwerkstruktur hinsichtlich hierarchischer Beziehungen zwischen den Knoten analysiert. Die daraus generierten Werte identifizieren 
zentrale Akteurinnen und Akteure aufgrund unterschiedlicher Merkmale. Anhand der Anzahl eingehender («Indegree») und ausgehender («Outdegree») Verbindungen wird die «structural importance of a node» (Borgatti, Everett, und Johnson 2018, 175) für den Informationsfluss innerhalb des Gesamtnetzwerks ermittelt. Zusätzlich zählen die strukturelle «Nähe» eines Knotens zu allen anderen Knoten, als sog. «Closeness» und die Vermittlerposition anhand der Anzahl kürzester Wege, die über einen bestimmten Knoten führen, die sog. «Betweenness》 zu den wichtigsten Werten, um Aussagen über die Wichtigkeit einzelner Knoten im Netzwerk zu generieren (Borgatti, Everett, und Johnson 2018, 183fff). Aufgrund der Fragestellung im Kontext der hier dargestellten Analyse wird speziell der Eingangsgrad betrachtet.

Der Eingangsgrad wird als Anzahl an Einladungen in Lerngruppen interpretiert, die eine Person erhalten würde. Die Anzahl eingehender Verbindungen zu einem Knoten wird innerhalb der analysierten Netzwerkstruktur als Teil der Bedeutungskonstitution aufgefasst. Die stellen eine Auswahl an Sinnangeboten zur Verfügung, welche Akteurinnen und Akteure in eigene sozial-kommunikative Strukturen integrieren, ablehnen, oder neu verhandeln.

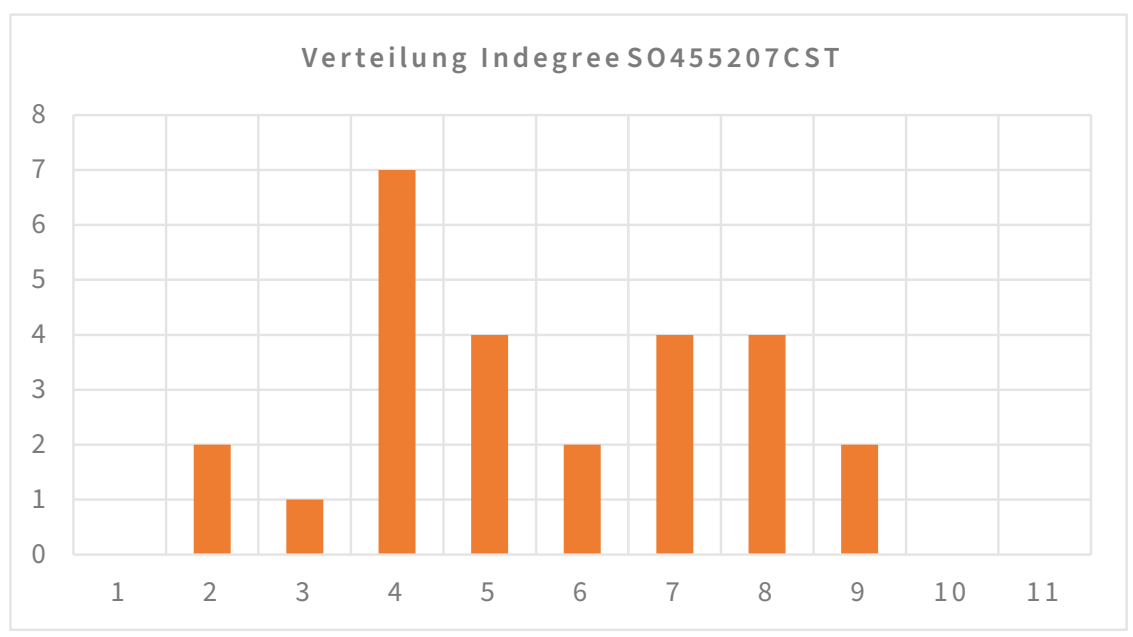

Abb. 2.: Verteilung Eingangsgrad SO455207CST (eigene Darstellung). 
Im Netzwerk SO455207CST streut die Anzahl der eingehenden Verbindungen zwischen 1 und 8 mit einem Mittelwert von 4.58, wobei die meisten Knoten 3, gefolgt von 4, 6 oder 7 eingehenden Verbindungen, aufweisen. Es gibt im vorliegenden Graphen keine isolierten Knoten (ohne Eingänge) und 12 von 26 Knoten weisen einen Eingangsgrad über dem Mittelwert auf, wobei die höchsten Eingangsgrade von Mädchen erzielt werden und die Werte 1 und 2 nur von Jungen. Grundsätzlich sind die Verbindungen innerhalb des Netzwerks gleichmässig verteilt und es gibt keine Knoten, die auffällig viele Verbindungen auf sich konzentrieren.

Neben dem akteursbezogenen Eingangsgrad können strukturelle Aussagen über die Netzwerkstruktur getroffen werden. Ein Beispiel sind die sogenannten «Modularitätsklassen». Diese erlauben die Identifikation von Teilgruppen innerhalb des Netzwerkgraphen. Diese Klassifizierung dient der strukturellen Analyse der Beziehungen zwischen den Pfaden. In der mathematischen Beschreibung und simultanen Visualisierung der Netzwerkstruktur werden mehrere analytische Schritte parallel ausgeführt. Erstens wird die strukturelle «Nähe» zwischen den Knoten über ein Anziehungs-Abstossungs-Prinzip ermittelt. Verbundene Knoten werden in einem mehrschrittigen Verfahren (Blondel u. a. 2008, 4ff) zu Clustern zusammengezogen. Alle diese Schritte resultieren in der grafischen Darstellung der Netzwerkstruktur, was anhand der Visualisierung des Netzwerks SO55207CST (s. Abb. 3) gezeigt wird. Der Graph enthält 26 Knoten, insgesamt liegen aus der Klasse Netzwerkdaten von 25 Schülerinnen und Schülern vor. Knoten von Mädchen werden violett, jene von Jungen grün und ohne Angabe des Geschlechts orange dargestellt: 


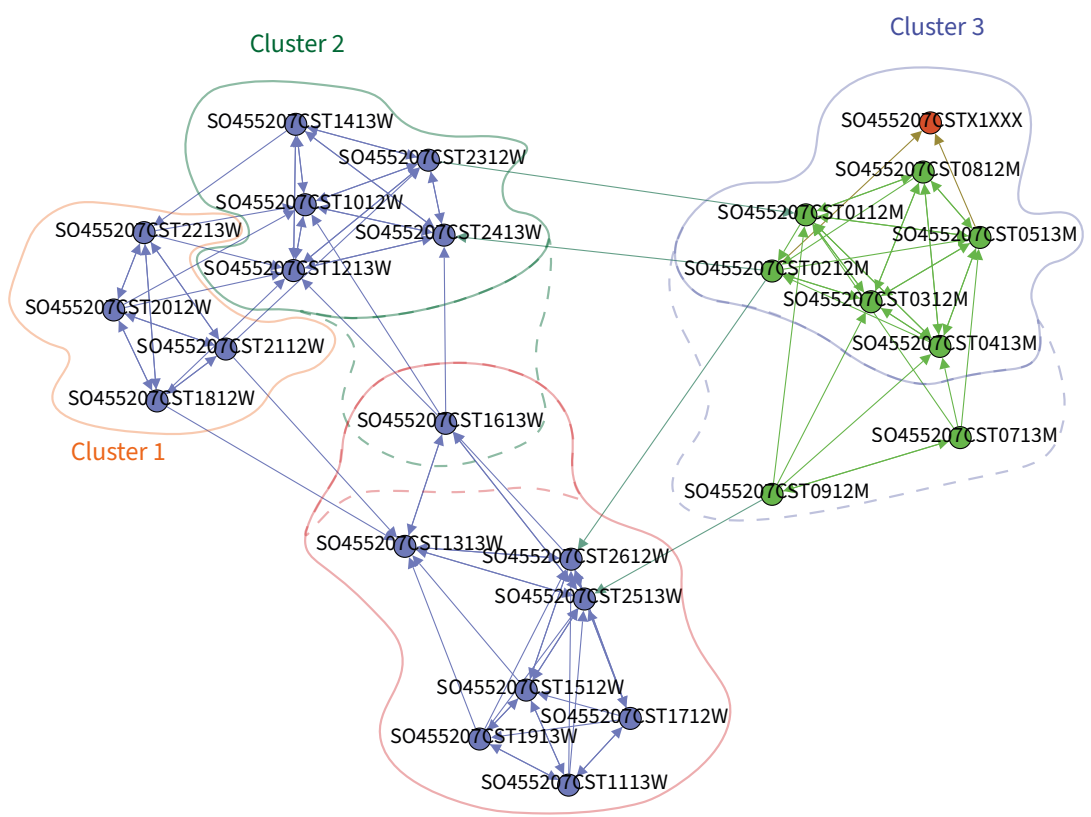

Abb. 3.: Darstellung Cluster SO455207CST (eigene Darstellung).

In Abbildung 3 ist die Zuteilung der einzelnen Knoten in Teilgruppen, sog. Cluster, zu erkennen. Grenzfälle und strukturelle Unschärfen können nun geklärt werden. Knoten ohne eingehende Verbindungen werden aus der Teilgruppe exkludiert (originale Zuteilung anhand Schraffierung). Innerhalb der Schulklasse SO455207CST lassen sich vier Cluster identifizieren. Aufgrund der Anzahl reziproker Verbindungen ist die Wahrscheinlichkeit, dass ein oder mehrere Lerngruppenchats existieren, innerhalb dieser Häufungen am grössten.

Im Anschluss an die Netzwerkstruktur der Schulklasse geht es darum, die Relationen in ihren kommunikativen Zusammenhang zu stellen. Die Analyse der Motive für die WhatsApp-Gruppen wurde als offene Frage formuliert, deren Antworten interpretativ ausgewertet wurden. 


\section{2 «Hausaufgaben», «lernen» und «einfach über alles schreiben» - Ergebnisse der interpretativen Auswertung}

Die Auswertung der Motive für die WhatsApp-Gruppen erfolgte mehrschrittig nach dem Vorbild der Grounded Theory Methodologie. Auf ein offenes Codieren folgte dimensionalisierendes Codieren, bzw. «focused coding» (Charmaz und Belgrave 2015, 3). Der iterative Prozess zwischen Einzelantwort und Codesystem brachte erste Konzepte hervor, die innerhalb zweier unabhängiger Interpretationsgruppen diskutiert und anschliessend überarbeitet wurden. Die Überarbeitung resultierte in vier Dimensionen, die hier als erstes, nicht abschliessendes Ergebnis vorgestellt werden:

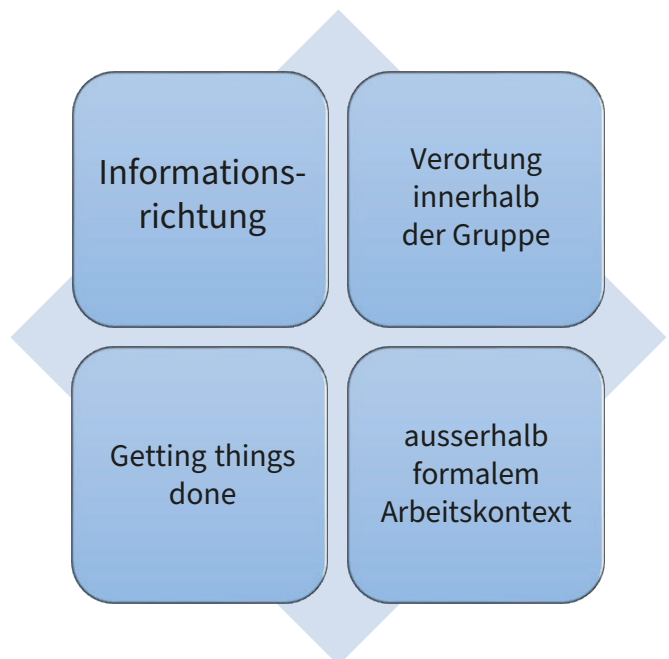

Abb. 4.: Dimensionen (eigene Darstellung).

- Die Informationsrichtung gibt Hinweise darauf, ob Informationen aus der Gruppe erhalten, an die anderen gegeben werden sollen, oder ob es sich um eine Wechselseitigkeit im Sinne eines «gegenseitig helfen» (SO455207CST2012W) handelt.

- Mit Formulierungen wie «Wir» oder «Ich» findet eine Verortung innerhalb oder gegenüber der Gruppe statt. Interessant sind hier die Formulierungen «jemand», also der Hinweis auf eine unbestimmte Einzelperson, bzw. das Adverb «man» als vage Generalisierung. 
- «Getting things done» bezeichnet den Workflow in der Lerngruppe sowohl den Inhalt betreffend als auch bezogen auf Arbeitsformen. Thematische Bezüge sind Hausaufgaben und Schulfächer im allgemeinen und Sharing, beispielsweise von Fotos des Materials, sowie die gemeinsame Organisation des Schulalltags.

- Einige Antworten verweisen auf soziale Aktivität ausserhalb des schulischen/formalisierten Lernkontext und lassen die Grenzen zwischen schulischen Inhalten und privater, informeller Konversation verschwimmen. Beispielsweise kann «Schreiben» als soziale Aktivität interpretiert werden. Durch das «einfach über alles schreiben» (BE311006SBO0812W) wird eine kommunikative Kopräsenz erzeugt, die den Austausch über den Inhalt stellt. Unbestimmte Angaben wie «Alles ein bisschen» (SZ883208BSCo614M), sowie Nennungen alternativer Lernkontexte, wie gemeinsames Gamen (Videospiele), oder Videos schauen, verweisen auf soziale Aktivitäten innerhalb des Klassenverbandes abseits formalisierter Lernaktivitäten.

Auf der Bedeutungsebene findet eine Sinnkonstruktion für die Lerngruppe aus Perspektive der Einzelnen und in Relation zum Kollektiv der Schulklasse statt. Die Motive können als Inhalte der Kanten im Netzwerk interpretiert werden. Als Beziehungsinhalt (Hollstein 2010, 464) bezeichnen sie die individuellen Zuschreibungen an die Lerngruppen. Eine simultane Analyse der Netzwerkstrukturen und Motive gibt anschliessend einen Einblick in die Beziehungen zwischen Gruppenstrukturen und thematischen Verortungen. Anhand eines Beispiels aus SO455207CST wird im nächsten Abschnitt die Verbindung der Netzwerkstruktur mit der Bedeutungsebene illustriert. 


\subsection{Netzwerkdaten und Bedeutungskonstitution verbinden - Ergebnisse der Mixed-Methods Analyse}

Die Codierung der Motive bewirkte im Zuge der Dimensionalisierung eine Entkoppelung von den konkreten Handlungszusammenhängen, auf welche sie sich beziehen. Aus diesem Grund wurden die Motive zusätzlich in ihrem jeweiligen Kontext betrachtet. Die Interpretation der Modularitätsklassen (s.o.) als «Cluster» ermöglicht eine strukturelle Analyse der Beziehungsinhalte, sowie deren weitere Verdichtung. Die codierten Antworten werden den Knoten im Netzwerk zugeordnet und in Beziehung zu ihren näheren Verbindungen und in weiterer Folge zum gesamten Netzwerk interpretiert. Die Darstellung der strukturellen Einbettung innerhalb der Schulklasse als Netzwerk rückt die thematischen Rahmungen ins Verhältnis zu einander und ermöglicht die Annäherung an gemeinsame Bedeutungskonstitutionen. Aus der relationalen Analyse lassen sich Aussagen treffen, die nur in den Netzwerkdaten oder allein der Auswertung von Motiven nicht generiert werden könnten. Erst die Verbindung beider Datenformen gibt Einblick in relationale Sinnkonstruktion.

\subsection{1 《Fragen über Fragen» oder «Peer Coaching》 - zwei Beispiele für thematische Rahmungen}

Als Beispiel werden zwei Cluster auf ihre relationalen Strukturen, Sinnkonstruktionen und Bedeutungszusammenhänge analysiert. In der Clusterdarstellung wurden die codierten Antworten den Knoten zugeordnet und nach den o.a. Dimensionen farblich markiert, wie in Abbildung 5 ersichtlich wird. 


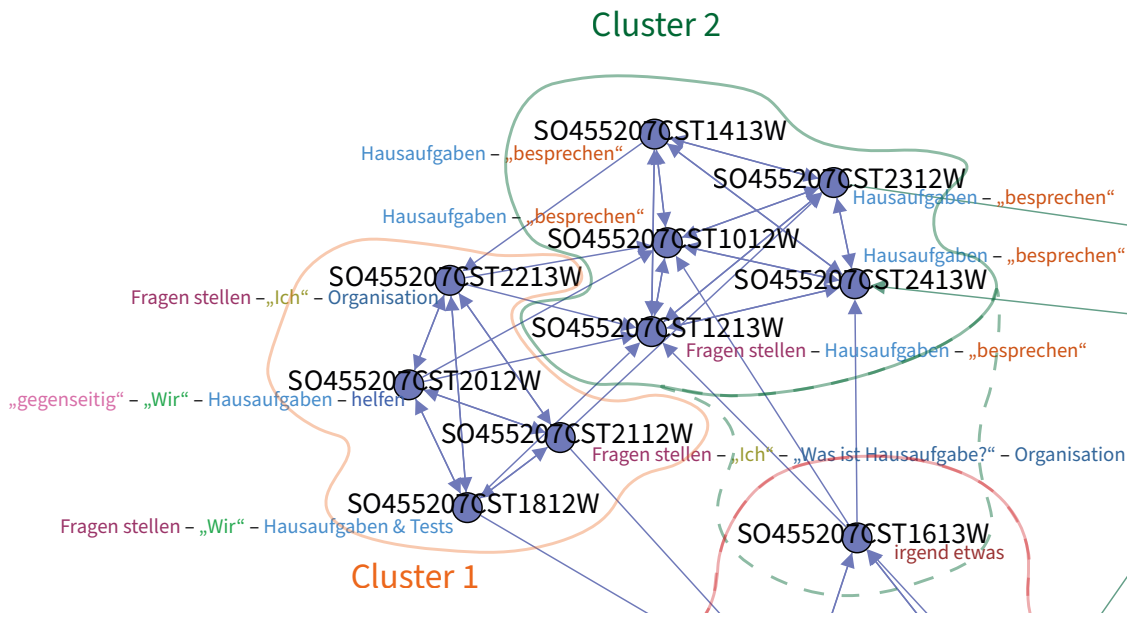

Abb. 5.: Cluster 1 \& 2 aus SO455207CST (eigene Darstellung).

Trotz unterschiedlicher Modularitätsklassen liegen die beiden Gruppen innerhalb des Graphen «nahe» beieinander. Die Schülerinnen aus Cluster 1 nennen jeweils einander und jede von ihnen hat mindestens eine ausgehende Verbindung zu einer Akteurin aus Cluster 2, das erklärt die grafische Nähe, da Knoten mit einer Verbindung zu einander sich im Graphen gegenseitig anziehen (s. 3.1). Auf die Verbindungen zu Cluster 4, kann im Rahmen der hier fokussierten Analyse nicht eingegangen werden. Gemeinsam ist den Gruppenmitgliedern aus Cluster 1 neben der Orientierung an einander ein «Streben nach aussen». Die Schülerinnen konstruieren einen homogenen Kontext für gemeinsame Lernaktivitäten. Es geht um schulische Themen im Sinne von Hausaufgaben, Tests und organisatorischen Fragen. Bei zwei der Mädchen findet sich eine Verortung des Individuums als «Ich» in Abgrenzung vom «Wir» der beiden anderen. Fragt man nun nach Einbettung in den kollektiven Gestaltungskontext der Gruppe, haben sie gute Chancen auf Konsens. Diese Homogenität birgt allerdings das Problem, dass mindestens eine von ihnen die Antworten auf die Fragen wissen müsste, die sie alle stellen möchten. Das kann dazu führen, dass die Subgruppe keine geeignete Ressource für die benötigte Hilfe oder Vergewisserung darstellt. Für SO455207CST2012W ist es aufgrund der Gruppenstruktur vermutlich nicht leicht, ein Klima des «gegenseitig Helfens» zu etablieren. Es bleibt offen, ob die Gruppe gemeinsam Wege findet, auf 
ihre Fragen Antworten zu suchen und sich kollektive Informationskompetenz anzueignen. Ob es überhaupt zu einer Zusammenarbeit kommt, oder ob am Ende «Fragen über Fragen» übrigbleiben, muss aufgrund der Datenlage vorerst offenbleiben. Ausgehend von der Problematik, die eine solche Homogenität mit sich bringt, die in einer Desintegration münden kann, lohnt es sich aufgrund der Ähnlichkeiten in der Vernetzungsstruktur, auf Cluster 2 zu blicken.

Die Akteurinnen aus Cluster 2 sind ebenfalls eng verbunden, im Unterschied zur anderen Gruppe hat nur SO455207CST1413W eine ausgehende Verbindung, die sie - nicht reziprok - mit SO455207CST2213W aus Cluster 1 verbindet. Die Schülerinnen konstruieren ein Lernumfeld, das hoch effizient anmutet. Sie antworten homogen und in Bezug auf Hausaufgaben als einziges Thema. Alle fünf Mädchen bezeichnen ihren Austausch in der Gruppe als «besprechen». Diese Formulierung deutet auf eine Vermischung von alltäglicher Peer-Kommunikation mit schulischen Aktivitäten und eine Gleichrangigkeit der Akteurinnen hin. Ein gegenseitiges «PeerCoaching» steht über Hilfe- oder Informationsgewinn aus der Gruppe.

Nur eine Akteurin aus Cluster 2 möchte Fragen stellen. Im Gegensatz zu SO455207CST2012W hat sie gute Chancen, dass ihr Interesse an Information aus der Gruppe in der generellen Wechselseitigkeit aufgefangen wird. Sie ist in eine Gruppe eingebettet, die Orientierung an gemeinsamer Arbeit in den Vordergrund stellt. Für die Akteurinnen aus Cluster 1 erscheint es erstrebenswert, Teil dieser Gruppe zu sein, wie die ausgehenden Verbindungen zeigen. Das könnte auf ein klasseninternes Wissen darüber hinweisen, dass Cluster 2 effektiv zusammenarbeitet, oder dass in dieser Gruppe Peer-Aktivitäten stattfinden, die für die anderen Mädchen interessant sind. Cluster 2 stellt eine fast abgeschlossene Clique dar. Dieses Merkmal kann in Bezug auf Ausgrenzung diskutiert werden. Die Gruppe scheint zwar intern effizient zu sein, aber nicht daran interessiert, ihre Ressourcen und Arbeitsweisen ausserhalb zu teilen. Hier deuten sich Fragen nach Teilhabe und Ausschluss als soziale Dimensionen, bzw. als weitere Analyserichtung an.

Auffällig an beiden Clustern ist Homogenität, die in unterschiedliche Richtungen weisen kann. Erste Schlüsse aus der iterativ-rekonstruktiven Codierung werden im nächsten Punkt diskutiert. 


\section{Interpretation der Ergebnisse und Ausblick}

Die Bildung von Lerngruppen gründet nicht nur auf der gemeinsamen Erledigung schulischer Aufgaben. Es deuten sich soziale Dynamiken an, die über inhaltliche Gemeinsamkeiten hinaus gehen. Sozialer Anschluss wird über gemeinsame Kommunikationsformen, vor allem aber über thematische Rahmungen konstruiert.

Die Mädchen aus Cluster 1 und Cluster 2 im Beispiel oben zeichnen sich durch gruppeninterne Homogenität bezüglich ihrer thematischen Anliegen aus. Diese ist geprägt von gemeinsamer schulbezogener Aktivität. Kommt das soziale Element des «Besprechens» hinzu, ergibt sich eine Zusammenarbeit auf Augenhöhe, die es auch einer Akteurin mit Fragen ermöglicht, innerhalb ihrer Clique Antworten und Vergewisserung zu erhalten. Die meisten Akteurinnen in Cluster 1 wollen Informationen aus der Gruppe bekommen. Dies kann dazu führen, dass die Lerngruppe ineffizient ist, wenn sie lediglich unter sich bleibt. Antworten allerdings die Akteurinnen aus Cluster 2 auf die Einladungen aus Cluster 1, so kann daraus eine Erweiterung der Perspektiven entstehen. Die strenge Homogenität von Cluster 2 kann durch andere Aspekte gemeinsamen Lernens erweitert werden. Cluster 1 könnte durch die Integration der Kommunikation auf Augenhöhe in Cluster 2 zu selbstständiger Informationskompetenz gelangen. Das Gruppenmotiv «Fragen stellen» könnte über eine Kombination der Anliegen aller Gruppenmitglieder aus beiden Gruppen in gemeinsame Informationssuche gewandelt werden. Hier zeigt sich die konstruktive Rolle von Akteurinnen, die andere Sichtweisen entwickeln und einbringen. In Bezug auf Agency und Structure lässt sich anhand dieser Formationen zeigen, wie individuelle Handlungsmöglichkeiten mit kollektiven Dynamiken verbunden sind. Gegenseitige Abhängigkeiten, soziale Regeln und Mechanismen von Teilhabe sind innerhalb der Cluster und im Klassenverband wirksam. Trotzdem ist jede Schülerin in der Lage, Sinnkonstruktion zu leisten und sich in ihrem eigenen Medien- und Lernhandeln aktiv in der Bedeutungsstruktur zu bewegen. Letztlich sind in diesen Handlungskontexten Hinweise auf Potentiale von Medienbildungsverhältnissen zu finden: 
- Erstens durchdringen Schülerinnen und Schüler die Komplexität der sozialen Struktur und gestalten das Verhältnis zu den anderen über ihr kommunikatives Handeln.

- Zweitens repräsentieren sie innerhalb der Lerngruppe ihren individuellen Kontext vermittels Artikulationen. Diese sind zugleich Reflexion auf eigenes Handeln und auf ihr eigenes Selbstverhältnis.

- Drittens verhalten sie sich zu den Dingen in der Welt, über Aneignung des Mediums als Artefakt innerhalb der Struktur und über die Aneignung spezifischer Kommunikationsformen.

Die Interpretation der Gruppenbildungen und Motive für die entsprechenden WhatsApp-Gruppen zeigt, dass diese, wie oben erwähnt, gleichzeitig auf konstruierte, etablierte Strukturen und konstruierende Aushandlungsprozesse im Klassenverband verweisen. Das gegenseitige Fragenstellen im Beispiel oben bringt als gemeinsame Kommunikationsform die Gruppe von Mädchen zusammen und ist thematischen Rahmung für diese Gruppenbildung. Die gruppeninternen sozialen Prozesse sind insofern geprägt von gemeinsamen Anliegen und der Art und Weise, wie diese kommunikativ verhandelt werden. Zugleich ermöglicht, bzw. verhindert die soziale Struktur der Schulklasse Teilhabe an anderen Gruppen. Die integrative Analyse der Sinnzuschreibungen gemeinsam mit der Darstellung relationaler Struktur wirft ein differenziertes Bild auf das Medienhandeln innerhalb der Schulklasse als bedeutsamer sozialer Formation in informellen Lernkontexten. Weitere Fragen, die sich daraus ergeben, sollen folgend in einem Ausblick auf die weitere Analyse und Datenerhebung diskutiert werden.

\section{Ausblick}

Die oben dargelegte Analyse ist der Anfang einer Suchbewegung, mediales Handeln in Schulklassen auf unterschiedlichen Ebenen zu erfassen und in einem theoretischen Modell abzubilden. Sozial konstruierte und kommunikativ ausgehandelte Lernaktivitäten auf Basis der Selbstverortung, der Informationsrichtung, der thematischen Rahmung und des Hinweises auf Verbindungen mit ausserschulischen Peer-Aktivitäten sind 
Hypothesen, die es an weiterem Material zu prüfen gilt. Aus den bisherigen Ergebnissen deuten sich weitere Analyseschritte an.

Das Wissen um Gruppenbildungen innerhalb der Klassen als dynamische Strukturen und die Netzwerkdarstellung als Momentaufnahme, führt zum Anliegen, sie wieder in ihre Dynamik zurück zu führen. Die Entstehung und weitere Entwicklung der Lernchats in WhatsApp und die dabei stattfindenden Aushandlungsprozesse können anhand der aktuell verfügbaren Daten noch nicht dargestellt werden.

Weiters weist die relationale Analyse darauf hin, dass «Sharing» als Wissensweitergabe an soziale Mechanismen gebunden ist. Dies wirft die Frage auf, in welchem Verhältnis Sharing und Kollaboration zu Teilhabe als strukturelle Voraussetzung und Ressource für gemeinsame Lernaktivitäten stehen. Anhand der WhatsApp-Gruppen-Dynamiken kann exemplarisch ermittelt werden, wie Teilhabe in gemeinsamen Aushandlungsprozessen konstruiert wird.

Als zusätzliches Datenmaterial zur Verdichtung der Analyse werden Daten aus der Tagebuchstudie im Projekt «Hausaufgaben und Medienbildung» herangezogen. Personenbezogene Daten liefern Kontextinformationen über die einzelnen Schülerinnen und Schüler. Weiters werden die Journale systematisch auf Nennungen von Kolleginnen und Kollegen, Nachrichteninhalte, und Hinweise auf (einen) Klassenchat(s) durchsucht, sowie als weiterer Fokus Einträge zu Hausaufgabenaktivitäten in Zusammenarbeit mit Kolleginnen und Kollegen codiert. Zusätzlich zur Codierung der Medientagebücher ist eine weitere Erhebungsphase auf Basis der weiterführenden Fragestellungen geplant.

Bisher kann festgehalten werden, dass thematische Rahmungen und Akteurskonstellationen innerhalb der Schulklassen die Gruppenstrukturen und ihre Sinnangebote beschreibbar machen. Der Analyserahmen und die theoretischen Verortungen müssen im weiteren Verlauf des Projekts weiter hinterfragt werden. Es werden Themen einbezogen wie zum Beispiel schülergenerierte Contexte [sic.] (Seipold 2017) als Verweisungszusammenhang zwischen Schulthemen und Lernaktivitäten sowie alltäglichem Medienhandeln. Die Herausforderung besteht weiterhin darin, Konzepte zu entwickeln, um diesen Phänomenen auf Ebene der Theoriebildung zu begegnen. 


\section{Literatur}

Archer, Margaret Scotford. 2000. Being Human: The Problem of Agency. Cambridge: Cambridge University Press.

Asterhan, Christa S. C., und Edith Bouton. 2017. «Teenage peer-to-peer knowledge sharing through social network sites in secondary schools». Computers \& Education 110 (Juli): 16-34. https://doi.org/10.1016/j.compedu.2017.03.007.

Avci, Hulya, und Tufan Adiguzel. 2017. «A Case Study on Mobile-Blended Collaborative Learning in an English as a Foreign Language (EFL) Context». The International Review of Research in Open and Distributed Learning 18 (7). https:// doi.org/10.19173/irrodl.v18i7.3261.

Bachmair, Ben. 2009. Medienwissen für Pädagogen: Medienbildung in riskanten Erlebniswelten. 1. Aufl. Lehrbuch. Wiesbaden: VS, Verl. für Sozialwiss. https://doi. org/10.1007/978-3-531-91391-9.

Bachmair, Ben, John Cook, und Norbert Pachler. 2014. «Mobile Medien als Kulturressourcen für Lernen, ein kulturökologischer Beitrag zur Medienbildung». In Perspektiven der Medienbildung, herausgegeben von Winfried Marotzki und Norbert Meder, 209-33. Wiesbaden: Springer Fachmedien Wiesbaden. https:// doi.org/10.1007/978-3-658-03529-7_10.

Blondel, Vincent D., Jean-Loup Guillaume, Renaud Lambiotte, und Etienne Lefebvre. 2008. "Fast unfolding of communities in large networks». Journal of statistical mechanics: theory and experiment 2008 (10): P10008. https://doi. org/10.1088/1742-5468/2008/10/P10008.

Borgatti, Stephen P., Martin G. Everett, und Jeffrey C. Johnson. 2018. Analyzing Social Networks. 2nd edition. London: SAGE Publications Ltd.

Borgatti, Stephen P., und Daniel Halgin. 2011. «On Network Theory». SSRN Electronic Journal. https://doi.org/10.2139/ssrn.2260993.

Bouhnik, Dan, und Mor Deshen. 2014. «WhatsApp Goes to School: Mobile Instant Messaging between Teachers and Students». Journal of Information Technology Education: Research 13: 217-31. https://doi.org/10.28945/2051.

Burkitt, Ian. 2015. "Relational agency: Relational sociology, agency and interaction». European Journal of Social Theory 19 (3): 322-39. https://doi. org/10.1177/1368431015591426.

Burkitt, Ian. 2018. «Relational Agency». In The Palgrave Handbook of Relational SociologY, herausgegeben von François Dépelteau, 523-38. Cham: Springer International Publishing. https://doi.org/10.1007/978-3-319-66005-9_26.

Castells, Manuel. 2004. Das Informationszeitalter: Der Aufstieg der Netzwerkgesellschaft. Bd. 1. 3 Bd. UTB 8259. Opladen: Leske + Budrich.

Cetinkaya, Levent. 2017. «An Educational Technology Tool That Developed in the Natural Flow of Life among Students: WhatsApp». International Journal of Progressive Education 13 (2): 29-47. http://ijpe.penpublishing.net/makale/234. 
Cetinkaya, Levent. 2017b. «The Impact of WhatsApp Use on Success in Education Process》. International Review of Research in Open and Distributed Learning 18 (7): 59-74. https://doi.org/10.19173/irrodl.v18i7.3279.

Charmaz, Kathy C. 2011. «Den Standpunkt verändern: Methoden der konstruktivistischen Grounded Theory». In Grounded Theory Reader, herausgegeben von Günter Mey und Katja Mruck, 181-205. Wiesbaden: VS Verlag für Sozialwissenschaften. https://doi.org/10.1007/978-3-531-93318-4_9.

Charmaz, Kathy C., und Linda Liska Belgrave. 2015. "Grounded Theory». In The Blackwell Encyclopedia of Sociology, herausgegeben von George Ritzer. Oxford, UK: John Wiley \& Sons, Ltd. https://doi.org/10.1002/9781405165518.wbeosg070.pub2.

Elias, Norbert. 1999. Über den Prozeß der Zivilisation. Soziogenetische und psychogenetische Untersuchungen. Bd. 159. Suhrkamp Taschenbuch Wissenschaft. Berlin: Suhrkamp. https://books.google.ch/books?id=IJ-WtAEACAAJ.

Emirbayer, Mustafa, und Ann Mische. 1998. «What Is Agency?» American Journal of Sociology 103 (4): 962-1023. https://doi.org/10.1086/231294.

Feierabend, Sabine, Thomas Rathgeb, und Theresa Reutter. 2018. «JIM-Studie Jugend, Informtaion, Medien. Basisuntersuchung zum Medienumgang 12- bis 19-jähriger». https://www.mpfs.de/fileadmin/files/Studien/JIM/2018/Studie/ JIM_2018_Gesamt.pdf.

Giddens, Anthony. 1984. The constitution of society: outline of the theory of structuration. Cambridge: Polity Press.

Gimranova, Aliya, Madina Nurmanova, und A. S. CohenMiller. 2017. «Texting to Motivate Language Learning: WhatsApp Group Chats and Near Peer Role Modeling (NPRM)». LEARNING LANDSCAPES 11 (1): 121-35. https://doi. org/10.36510/learnland.v11i1.927.

Hayes, Aneta L., und Nasser Mansour. 2017. «Confidence in the Knowledge Base of English Language Learners Studying Science: Using Agency to Compensate for the Lack of Adequate Linguistic Identity». Research in Science Education 47 (2): 353-71. https://doi.org/10.1007/s11165-015-9504-8.

Hepp, Andreas. 2013. «Medienkultur als die Kultur mediatisierter Welten». In Medienkultur: Die Kultur mediatisierter Welten, herausgegeben von Andreas Hepp, 63-89. Wiesbaden: VS Verlag für Sozialwissenschaften. https://doi. org/10.1007/978-3-531-19933-7_4.

Hepp, Andreas. 2018. «Von der Mediatisierung zur tiefgreifenden Mediatisierung». In Kommunikation - Medien - Konstruktion. Braucht die Mediatisierungsforschung den Kommunikativen Konstruktivismus?, herausgegeben von Jo Reichertz und Richard Bettmann, 27-45. Wissen, Kommunikation und Gesellschaft. Schriften zur Wissenssoziologie. Wiesbaden: Springer Fachmedien Wiesbaden. https://doi.org/10.1007/978-3-658-21204-9_2.

Hepp, Andreas, Andreas Breiter, und Uwe Hasebrink, Hrsg. 2018. Communicative Figurations. Cham: Springer International Publishing. https://doi. org/10.1007/978-3-319-65584-0. 
Hepp, Andreas, und Uwe Hasebrink. 2014. «Kommunikative Figurationen - ein Ansatz zur Analyse der Transformation mediatisierter Gesellschaften und Kulturen». In Von der Gutenberg-Galaxis zur Google-Galaxis. Alte und neue Grenzvermessungen nach 50 Jahren DGPuK, herausgegeben von Nikolaus Jackob, Birgit Stark, und Oliver Quiring, 343-6o. Konstanz: UVK Verlagsgesellschaft.

Hepp, Andreas, und Friedrich Krotz. 2012. «Mediatisierte Welten: Forschungsfelder und Beschreibungsansätze - Zur Einleitung». In Mediatisierte Welten, herausgegeben von Friedrich Krotz und Andreas Hepp, 7-23. Wiesbaden: VS Verlag für Sozialwissenschaften. https://doi.org/10.1007/978-3-531-94332-9_1.

Hollstein, Betina. 2010. «Qualitative Methoden und Mixed-Method-Designs». In Handbuch Netzwerkforschung, herausgegeben von Christian Stegbauer und Roger Häußling, 459-70. Wiesbaden: VS Verlag für Sozialwissenschaften. https://doi.org/10.1007/978-3-531-92575-2_40.

Jannari, Diyana, Zaleha Abdullah, Hasnah Mohamed, Norasykin Mohd Zaid, und Baharuddin Aris. 2017. «Social Media in Learning: Insights of High Schools». ADVANCED SCIENCE LETTERS 23 (8): 7477-81. https://doi.org/10.1166/ asl.2017.9502.

Keogh, Conor. 2017. «Using WhatsApp to Create a Space of Language and Content for Students of International Relations». Latin American Journal of content \& language integrated learning 10 (1): 105-32. https://doi.org/10.5294/laclil.2017.10.1.4.

Krotz, Friedrich. 2007. Mediatisierung. Fallstudien zum Wandel von Kommunikation. Wiesbaden: VS Verlag für Sozialwissenschaften. https://doi.org/10.1007/9783-531-90414-6.

Krotz, Friedrich, und Andreas Hepp, Hrsg. 2012. Mediatisierte Welten. VS Verlag für Sozialwissenschaften. https://doi.org/10.1007/978-3-531-94332-9.

Meder, Norbert. 2007. "Theorie der Medienbildung. Selbstverständnis und Standortbestimmung der Medienpädagogik». In Jahrbuch Medien-Pädagogik 6: Medienpädagogik - Standortbestimmung einer erziehungswissenschaftlichen Disziplin, herausgegeben von Werner Sesink, Michael Kerres, und Heinz Moser, 55-73. Wiesbaden: VS Verlag für Sozialwissenschaften. https://doi. org/10.1007/978-3-531-90544-0_3.

Meder, Norbert. 2014. «Das Medium als Faktizität der Wechselwirkung von Ich und Welt (Humboldt)». In Perspektiven der Medienbildung, herausgegeben von Winfried Marotzki und Norbert Meder, 45-69. Wiesbaden: Springer Fachmedien Wiesbaden. https://doi.org/10.1007/978-3-658-03529-7_3.

Meder, Norbert. 2015. «Neue Technologien und Erziehung/Bildung». medienimpulse. Beiträge zur Medienpädagogik I (Medienpädagogik und E-Learning): 12.

Pachler, Norbert, Ben Bachmair, und John Cook. 2010. Mobile Learning. Structures, Agency, Practices. New York: Springer. https://doi.org/10.1007/978-1-44190585-7. 
Rosenberg, Hananel, und Asterhan, Christa S. C. 2018. ""WhatsApp, Teacher?" Student Perspectives on Teacher-Student WhatsApp Interactions in Secondary Schools». Journal of Information Technology Education: Research 17: 205-26. https://doi.org/10.28945/4081.

Rummler, Klaus. 2018. «Hausaufgaben und Medienbildung. Eine explorative Studie zur Ökologie des Medienhandelns im häuslichen Lernkontext von Sekundarschülerinnen und -schülern in der Deutschschweiz». Herausgegeben von Jasmin Bastian, Tobias Feldhoff, Marius Harring, und Klaus Rummler. MedienPädagogik: Zeitschrift für Theorie und Praxis der Medienbildung 31 (Digitale Bildung): 143-65. https://doi.org/10.21240/mpaed/31/2018.05.22.X.

Rummler, Klaus, Colette Stingelin Schneider, und Caroline Grabensteiner. 2018. «Medientagebuch. Forschungsinstrument des SNF-Projektes «Hausaufgaben und Medienbildung/», Juli. https://doi.org/10.5281/ZENOD0.4282456.

Seipold, Judith. 2017. «Lernergenerierte Contexte. Räume für personalisiertes und selbstgesteuertes Lernen und Ideengeber für ein „Ökologiemodell von Aneignung"». In Jahrbuch Medienpädagogik 13: Vernetzt und entgrenzt - Gestaltung von Lernumgebungen mit digitalen Medien, herausgegeben von Kerstin Mayrberger, Johannes Fromme, Petra Grell, und Theo Hug, 29-43. Wiesbaden: Springer Fachmedien Wiesbaden. https://doi.org/10.1007/978-3-65816432-4_3.

Seipold, Judith, Klaus Rummler, und Julia Rasche. 2010. «Medienbildung im Spannungsfeld alltäglicher Handlungsmuster und Unterrichtsstrukturen». In Medienbildung in neuen Kulturräumen: Die deutschprachige und britische Diskussion, herausgegeben von Ben Bachmair, 227-41. Wiesbaden: VS Verlag für Sozialwissenschaften. https://doi.org/10.1007/978-3-531-92133-4_16.

So, Simon. 2016. «Mobile instant messaging support for teaching and learning in higher education». The Internet and Higher Education 31 (Oktober): 32-42. https://doi.org/10.1016/j.iheduc.2016.06.001.

Sonia M. Livingstone, und Julian Sefton-Green. 2016. The Class: Living and Learning in the Digital Age. Connected Youth and Digital Futures. New York: New York University Press.

Suter, Lilian, Gregor Waller, Jael Bernath, Céline Külling, Isabel Willemse, und Daniel Süss. 2018. "JAMES - Jugend, Aktivitäten, Medien - Erhebung Schweiz». Zürich: Zürcher Hochschule für Angewandte Wissenschaften. https://www.zhaw.ch/storage/psychologie/upload/forschung/medienpsychologie/james/2018/Ergebnisbericht_JAMES_2018.pdf.

Täube, Volker G. 2010. «Cliquen und andere Teilgruppen sozialer Netzwerke». In Handbuch Netzwerkforschung, herausgegeben von Christian Stegbauer und Roger Häußling, 397-406. Wiesbaden: VS Verlag für Sozialwissenschaften. https://doi.org/10.1007/978-3-531-92575-2_35.

Truschkat, Inga, Manuela Kaiser-Belz, und Vera Volkmann. 2011. «Theoretisches Sampling in Qualifikationsarbeiten: Die Grounded-Theory-Methodologie zwischen Programmatik und Forschungspraxis». In Grounded Theory Reader, herausgegeben von Günter Mey und Katja Mruck, 353-79. Wiesbaden: VS Verlag für Sozialwissenschaften. https://doi.org/10.1007/978-3-531-93318-4_16. 